Horizons philosophiques

\title{
Les problèmes philosophiques rencontrés dans le projet d'une herméneutique de la production filmique
}

\section{Alain Létourneau}

Volume 12, numéro 1, automne 2001

Langue : identité plurielle

URI : https://id.erudit.org/iderudit/801199ar

DOI : https://doi.org/10.7202/801199ar

Aller au sommaire du numéro

Éditeur(s)

Collège Édouard-Montpetit

ISSN

1181-9227 (imprimé)

1920-2954 (numérique)

Découvrir la revue

Citer cet article

Létourneau, A. (2001). Les problèmes philosophiques rencontrés dans le projet d'une herméneutique de la production filmique. Horizons philosophiques, 12(1), 136-152. https://doi.org/10.7202/801199ar d'utilisation que vous pouvez consulter en ligne. 


\section{Les problèmes philosophiques rencontrés dans le projet d'une herméneutique de la production filmique 1}

Si nous parlons d'une herméneutique de la production filmique, nous désignons par là le projet de développer un "art de l'interprétation" susceptible d'être appris et d'être enseigné, concernant en particulier les œuvres cinématographiques. C'est donc d'une compétence interprétative qu'il s'agit, dont nous pensons qu'elle peut s'apprendre et se développer. Ce n'est évidemment pas dire qu'il n'y aurait qu'une seule herméneutique du film qui soit possible; c'est plutôt à tout un ensemble diversifié de possibilités qu'il faut songer. II doit y avoir d'autres attitudes possibles que celle qui consiste à dénigrer ces œuvres et leur fréquentation et celle qui consiste simplement en une entreprise promotionnelle. II manque cruellement une via tertia qui arriverait à manifester le sens que des œuvres filmiques peuvent avoir pour nous.

Cette question en soulève toutefois d'autres. Quelle vision du médium cinéma ce projet suppose-t-il? On ne peut considérer ce média sans considérer aussi l'ensemble des médias de masse, et leur place dans la culture. II faut donc aussi s'interroger sur cette dernière : ce qui arrive à la culture sous la guise de la "mondialisation», le fait des cultures en évolution et les caractéristiques de la culture contemporaine. Ces questions sont sous-jacentes aux réflexions qui suivent.

Pour réaliser le projet qui figure au titre, trois séries de problèmes doivent être traités brièvement. Un premier groupe de problèmes est d'ordre éthique et politique. Un second groupe de problèmes est d'ordre culturel. Un troisième groupe est de nature proprement critique et herméneutique. Une double série de questions qui seraient susceptibles de donner un cadre à une pratique herméneutique concrète viendra conclure le propos.

\section{Problème éthique et politique}

Développer une approche herméneutique des productions filmiques, c'est forcément chercher à dépasser la passivité foncière d'un public captif de l'offre culturelle des grands empires médiatiques de production d'images. Il doit pouvoir être possible d'être des citoyens qui délibéront de

1. Communication présentée au congrès «Philosophie et mondialisation», Société de philosophie du Québec, Trois-Rivières, 24 février 2001. 
notre avenir et de nos choix avec d'autres. Et il n'y a pas de raisons de cantonner ce genre de préoccupation à la seule philosophie politique, ou à l'éthique appliquée. Au contraire, il est possible d'estimer qu'une revalorisation et une éducation des capacités interprétatives de nos concitoyens est susceptible de favoriser les choix conscients, la prise de distance réfléchie par rapport aux contenus véhiculés et l'appropriation critique, en fonction de choix personnels, de ce qui se véhicule dans ces contenus. Ceci revient à proposer que l'herméneutique sache elle-même se faire une herméneutique appliquée, en sélectionnant des matériaux pertinents. Elle ne peut toutefois accepter ce qui serait une forclusion esthétisante en reniant ses liens avec les problématiques éthiques et politiques.

Partons d'un constat très simple : nous n'allons pas changer le fait que la population, en particulier la jeune population, est nourrie culturellement d'images et de récits, d'ailleurs pas uniquement cinématographiques mais multiples, les différents médias audiovisuels étant de moins en moins cloisonnés les uns par rapport aux autres. Un professeur peut bien avoir les œuvres de Paul Valéry comme livre de chevet (pour ne donner qu'un exemple) et se retrouver en classe avec des jeunes impliqués dans des jeux de rôles, qui sortent au cinéma, se baladent sur Internet, téléchargent des documents ou des fichiers de musique, sinon sur Napster du moins sur d'autres sites moins exposés, etc. La question d'une herméneutique des contenus média, en particulier filmiques, intervient ici dans un contexte pédagogique.

Mais alors, ne retrouverons-nous pas l'opposition classique entre les défenseurs de "l'art pour l'art" et les défenseurs de «'art militant", l'art au service d'une cause? Pour moi, cette discussion est dépassée dans la mesure où il ne peut s'agir de plaider pour un art militant, et de ne retenir comme matériaux signifiants que ce qui fait preuve d'un engagement déterminé. Le propos sur la citoyenneté et sur la délibération démocratique se situe à un autre niveau que celui de l'analyse et de l'interprétation des contenus. Mais ceci étant dit, il n'y a pas non plus d'herméneutique neutre des œuvres d'art. Le cinéma artistique a bien sûr son droit de cité et représente une nécessité pour notre vie culturelle, et c'est dans un contexte foncièrement libéral qu'il convient d'interpréter les œuvres. Mais si notre position politique n'est pas réfléchie ou si nous déclarons ces questions impertinentes, au nom par exemple de l'autonomie de l'herméneutique des œuvres, nous risquerions simplement d'entériner la domination effective de certains modèles et de certains courants puissants de la production cinématographique mondiale. II faut sans doute favoriser, comme lieux d'interprétation, les œuvres qui manifestent le 
mieux la richesse du média filmique, tant au point de vue de la forme qu'au point de vue du contenu. Une approche des productions standardisées à l'école des recettes de l'industrie peut aussi être le lieu privilégié d'une éducation critique. Ceci étant dit, nous ne devons pas non plus tomber dans un manichéisme ou un simplisme, puisque c'est toute la production cinématographique qui a des contraintes importantes de financement, et qu'à ce titre elle doit pouvoir rencontrer un public. Et il est difficile de penser que, par exemple, Lars Von Trier aurait commis une trahison face à l'esprit de Dogme 95 en créant Dancer in the Dark: les compromis ne sont pas toujours faits au prix de l'inventivité.

\section{Les problèmes de type culturel}

\subsection{Les caractéristiques de la culture contemporaine}

La culture est certes un phénomène extrêmement complexe. Elle englobe les façons de faire, les manières de parler et de penser, ces habitudes, langages et conceptualités qui se manifestent dans l'action individuelle, mais aussi le réseau de médiations et les institutions qui structurent et organisent notre vie sociale: domaines économique, politique, juridique, scientifique, éducatif, artistique et "culturel» au sens étroit, et les communications requises à chacun de ces plans et bien entendu entre ces derniers. Cette description de la culture ne se veut certes pas intégrale et suffisante.

Notre contexte politique, à la fois libéral et démocratique, est marqué par une social-démocratie en crise de mondialisation et d'hyper-libéralisation, d'où les idées d'État post-national et d'associations politiques de niveau plus vaste que l'État qui viendraient répondre au plan politique à l'extension de plus en plus grande des entreprises multinationales, parce qu'il n'est pas question de renoncer à la vie démocratique. On le sait, c'est paradoxalement grâce à l'État-social, appelé aussi État-providence, que les sociétés avancées ont trouvé le moyen de réguler le capitalisme. Pendant les trente glorieuses, appelées par Eric J. Hobsbawm l'Âge d'or, ce dernier a pu donner satisfaction à des demandes spécifiques des travailleurs, ce qui a permis le consumérisme généralisé aux masses, chose qui ne s'était pas vue dans d'autres périodes de l'histoire?. Le cinéma s'était développé au début du siècle et avait donné lieu à une première phase de domination de Hollywood ${ }^{3}$. Ce fut dans ce contexte un médium de divertissement extrêmement populaire, et on se souviendra que le

2. Eric J. Hobsbawm, L'âge des extrêmes. Histoire du Court XXe siècle. Bruxelles, Éditions Complexe/Le Monde diplomatique, 1999 p. 349.

3. E. J. Hobsbawm, op. cit. Avant 1914, Hollywood avait le quasi-monopole du marché mondial du cinéma, p. 141. À compter des années 1930, Hollywood ne manqua pas de récupérer une partie des avant-gardes, p. 245. Dès après la Grande Crise, les américains dominaient déjà l'univers médiatique mondial, p. 263. 
développement de la télévision à partir des années 1950 a donné lieu à bien des craintes à l'effet d'une possible disparition du grand écran. Aujourd'hui l'industrie du cinéma est rentable, surtout pour les gros joueurs d'une industrie plus que jamais dominée par les Américains, même si la part des entrées en salle a décru globalement ${ }^{4}$. Le cinéma a permis de vérifier une tendance qui me semble générale : le phénomène de l'emboîtement des médias, c'est-à-dire de l'intégration dans de nouveaux médias de productions de forme différente au départ.

Afin de caractériser l'époque contemporaine, plutôt que de parler d'un âge postmoderne, expression qui ne prend son sens que par différenciation et opposition avec la modernité, ouvrant dès lors un litige avec cette dernière qui me semble peu intéressant, j'estime préférable de considérer notre époque comme l'age des médias de masse et de la communication $^{5}$. On s'aperçoit de deux choses, qui subsistent en fait en même temps : certes, les grands récits de légitimation, pour parler comme Lyotard, sont en crise, mais ils ne disparaissent pas, ils sont simplement transformés : par exemple, l'individualisme contemporain est dans la lignée de l'émancipation de l'individu amorcée avec la philosophie moderne, les lumières et le libéralisme. II en est la transformation sous un mode consumériste. Le même genre de raisonnement s'applique en ce qui concerne le statut du savoir : l'apologie de la performance pure qui caractériserait l'état du savoir selon Lyotard est en fait déjà présente à l'âge industriel; ce sont essentiellement les modalités de diffusion et d'organisation qui changent. Et l'Université ne disparaît pas d'avoir vu ses sources publiques de financement diminuées; elle risque simplement d'être mise davantage au service d'intérêts particuliers et spécifiques. Les phénomènes de l'individualisme et du groupisme, décrits par Lipovetsky, peuvent être admis sans être considérés sous le mode postmoderne ${ }^{6}$. L'élément central qu'ont réussi à repérer les postmodernistes, c'est-à-dire le remplacement des méta-récits par les micro-récits, correspond à une dominante de la narrativité et de l'imaginaire qui accompagne un certain retour du mythe sous mode déréalisé, lequel s'accomplit avant tout dans et par les médias de masse. Ceci n'a pas à être expliqué par un discours

4. Laurent Creton, Cinéma et marché. Paris, Armand Colin, 1995, p. 224, tableaux 3.2 et 3.3; p. 228, tableau 3.9. Le nombre de salles est en dimininution marquée depuis le début des années 1980; le cas de la France n'est sans doute pas exceptionnel. Mais la diffusion de films à la télévision et le chiffre d'affaires des éditeurs vidéo sont en hausse, p. 241 et 242 , tableaux 5.2 et 5.4 .

5. Hypothèse développée dans $A$. Létourneau, «La religion de l'imaginaire à l'époque des communications". Studies in Religion/Sciences religieuses, vol. 26 no. 3, 1997, 309-335.

6. Gilles Lipovetsky, Le crépuscule du devoir. L'éthique indolore des nouveaux temps démocratiques. Paris, Gallimard, 1992; L'ère du vide. Essai sur l'individualisme contemporain. Paris, Gallimard, 1983. 
pathétique sur la crise de la rationalité. Certes, il semble désormais impossible de réaliser le système global de la raison, ne semblent demeurer que des travaux rationnels très restreints par leur portée. De cette manière, nous pouvons relire les théoriciens postmodernistes comme des contributeurs à l'esthétique philosophique. Les espaces nouveaux de la rationalité, désormais éclatée et hyper-spécialisée, sont appelés à se mettre en réseau : il est évident que ce discours très populaire dans la sphère de la recherche doit lui-même beaucoup à la vulgate de l'interconnexion, dont on manque à remarquer les liens avec une problématique à la fois technique et communicationnelle au sens restreint et médiatique du terme.

Sans donc entériner le vocable du postmodernisme, nous pouvons dire que Lyotard et Lipovestky ont néanmoins repéré certaines caractéristiques importantes au point de vue sociologique : en particulier ce rôle central de l'imaginaire dans la culture consumériste d'aujourd'hui. À mon avis toutefois, de même que la télévision n'a pas fait disparaître le cinéma, l'ordinateur n'enlève pas le livre et la culture mass-médiatique à grande échelle ne fait pas disparaître les traditions critiques antérieures. De même, quoi qu'en disent certains, le journalisme de masse n'a pas éliminé le journalisme critique servant à entretenir et développer le débat public aux fins de la vie démocratique de nos cités7. Si donc il est exact de dire que la culture commune semble beaucoup plus axée sur la consommation d'images et de récits que sur l'apprentissage et le développement des habiletés argumentatives et réflexives, il ne faut pas y voir un prétexte à une démission ou à une disparition annoncée de la pensée rationnelle et argumentée. Mais si ce diagnostic sur la culture contemporaine est exact, c'est en assumant aussi les aspects esthétiques et imaginaires de la culture contemporaine que la philosophie comme discipline réflexive pourra de manière nouvelle manifester sa crédibilité et assurer son travail de raison. Bien sûr, ce travail de raison est aussi multiple que les figures de la raison et ses diverses modalités. Pour permettre toutefois le passage de la narration à l'argumentation, la discipline de l'herméneutique permet la transformation de l'image, des récits et du rêve commercialisés par l'industrie culturelle en contenus culturels discutables et argumentables.

7. Tradition qui remonte à la critique des industries culturelles de l'école de Francfort, relayée par le premier Habermas; of J. Habermas, L'espace public. Paris, Payot, nouvelle édition 1992, ouvrage paru en allemand en 1962. Dans la nouvelle Préface à cet ouvrage, Habermas a nuancé beaucoup, comme d'ailleurs dans Droit et démocratie, paru en allemand en 1992, ce qu'il disait alors du commercialisme des médias. Voir à ce sujet $A$. Létourneau, "Remarques sur le journalisme et la presse au regard de la discussion dans l'espace public", dans P. J. Brunet (dir.), L'éthique dans la société de l'information. Québec et Paris, Presses de l'Université Laval et l'Harmattan, 2001, p. 47-72. 
Nous pouvons certes parler aujourd'hui d'une culture consumériste qui se maintient après les années d'abondance et après les désillusions face au rêve de la "société de consommation" qui devait remplacer la société du travail. Le meilleur symbole de cette culture consumériste est encore l'évolution d'Internet : média développé d'abord dans un contexte militaire, puis réseau de chercheur, il ressemble de plus en plus à une sorte de supermarché virtuel. Nos jugements moraux parfois à l'emportepièce au sujet de cette culture de consommation ne nous empêchent d'ailleurs pas de faire partie, malgré tout, de cette culture consumériste d'une manière ou de l'autre. II y a d'ailleurs consommation et consumérisme, et la critique du conformisme est assez facile et puérile si elle ne propose pas d'alternatives rendant possible une meilleure participation des agents.

Nous avons tendance aussi à ne traiter du rapport aux médias qu'en termes de consommation : cotes d'écoute, parts de marché livrées aux publicitaires par les médias. Les études qui visent à vendre des produits n'ont pas d'autre but que d'agrandir leur efficacité et de mieux réussir à atteindre leurs objectifs de pénétration de marchés. D'où l'image classique d'un public passif, manipulé et influencé par les médias. De telles conceptions, sous-jacentes dans les premières mass media studies, minimisent le rôle des personnes en tant que citoyens, capables de sélection et aussi de critique réfléchie de tout ce qui est véhiculé dans les médias de masse, qu'il s'agisse de la télévision, du cinéma ou des autres médias ${ }^{8}$. II est possible de penser que le peu d'intérêt pour les causes communes, et le peu de participation des individus en général à la vie publique et à ses débats, est au fond la résultante d'un manque de prise au sérieux des nouvelles tâches d'éducation qui sont requises, entre autres, par le développement de cette culture de masse omniprésente aujourd'hui. En proposant des médiations herméneutiques rendant possible une réappropriation critique et réflexive des éléments véhiculés sous mode narratif, qu'il soit d'ailleurs visuel, audiovisuel ou simplement audiophonique, il deviendrait possible de relever le niveau critique et la participation civique. Ceci dans la mesure en particulier où l'expérience de l'analyse interprétative montre que rien n'est par là enlevé à la qualité du divertissement, bien au contraire, puisqu'à la participation privée du contenu média succéderaient des discussions critiques et une animation susceptible de rendre conceptuel et discursif ce qui se présente d'abord sous un mode narratif. Ceci pourrait être une piste pratique et pédagogique intéressante pour les philosophes, en particulier les professeurs.

8. Pensons en particulier à Harold $\mathrm{H}$. Lasswell concernant l'impact des médias. Cf. B. Miège, La pensée communicationnelle. Grenoble, PUG, 1995. 
Cela ne va toutefois pas sans d'importants problèmes critiques, dont il sera question plus loin.

\section{Notre archaïsme culturel}

Par «archaïsme culturel», il faut entendre quelque chose qui caractérise non pas simplement la philosophie comme discipline, mais bien l'ensemble de notre civilisation telle qu'elle s'est construite depuis les inscriptions sur la pierre et les manuscrits. Certes, nous sommes maintenant dans une culture des hyperliens, de ce que M. Vanderdorpe appelait la tabularité, mais nous n'avons pas encore opéré une conversion mentale complète compte tenu des héritages culturels plus anciens qui néanmoins nous marquent profondément ${ }^{9}$. C'est bien sûr de la domination du mode écrit que je veux parler, ce média si puissant qui a contribué à fixer et transmettre la pensée et la parole bien plus que ne pouvait le faire la simple voix humaine. Il a bien sûr vu sa puissance démultipliée par l'invention de l'imprimerie, qui a connu encore de fabuleux développements à l'époque contemporaine. II se prolonge non seulement par la radio et la télévision, mais encore par Internet dans la mesure où l'écrit y est omniprésent.

La philosophie en particulier s'est développée beaucoup en tant que commentaire de textes. Elle est traditionnellement la discipline des questions profondes, mais comme Deleuze nous a appris que le superficiel est le plus profond, il ne faut pas trop se fier à ce genre d'opposition topique. Certes, la productivité théorique des philosophes demeure importante aujourd'hui, et elle est conduite à une spécialisation de plus en plus grande, qui se voit de manière évidente. Bien peu de philosophes ont consacré du temps et de l'énergie à penser les nouveaux médias. L'oscillation se joue en général entre deux extrêmes, la dénonciation unilatérale au profit de l'écrit ou la célébration de ces nouvelles versions de la noosphère dont parlait Teilhard de Chardin, qui s'appellent aujourd'hui cyberespace, conscience planétaire ou intelligence collective chez Pierre Lévy, et autrement par Joël de Rosnay avec le cybionte, Derrick de Kerkhove, etc. ${ }^{10}$. II est quand même symptomatique que l'on arrive à voir dans la mise en réseau que permet une série d'ordinateurs interconnectés quelque chose qui ressemble à la conscience collective

9. Christian Vanderdorpe, Du papyrus à l'hypertexte. Essais sur les mutations du texte et de la lecture. Montréal, Éd. Boréal, 1999. La tabularité, c'est le lien entre les textes, qui nous permet de sauter de l'un à l'autre comme on zappe à la télévision.

10. Dans cette veine, voir la traduction récente de l'ouvrage du titulaire du programme McLuhan à l'Université de Toronto. Derrick de Kerckhove, L'intelligence des réseaux. Paris, Odile Jacob, 2001. Mentionnons aussi Doug Rushkoff avec l'idée de "cerveau planétaire". Des lectures plus sobres de ce phénomène semblent non seulement possibles mais souhaitables. 
chez Durkheim : un postulat d'unité de la société, alors qu'un Mead plus modeste parlait plutôt de l'Autrui généralisé11. Souhaitant réfléchir sur le médium visuel et filmique en particulier, le modèle du commentaire de texte est prégnant, parce qu'au fond des auteurs auraient indiqué des pistes théoriques incontournables pour aujourd'hui. Nous devons avoir parfois le courage de poser nous-mêmes les questions et de tenter d'y apporter réponse. Bien que considérant que nous devons prendre en compte en herméneutique les travaux de Habermas, de Gadamer, de Eco et de Ricœur, l'herméneutique pourrait et devrait s'accomplir non pas uniquement dans le commentaire de ces auteurs (et même de Blondel), mais tout autant dans l'interprétation effective des œuvres ${ }^{12}$. Le domaine de "l'herméneutique philosophique" souffre de ne pas assez dépasser la théorie herméneutique ou le commentaire de textes d'auteurs réputés.

Compte tenu de l'hyper-spécialisation qui atteint toutes les sphères de la production scientifique y compris la philosophie, il y a une illisibilité du texte philosophique qui ne favorise pas son accessibilité. Ce sont les spécialistes du langage afférent qui arrivent à y comprendre quelque chose : les spécialistes de la phénoménologie s'adressent à leurs collègues, les spécialistes de la pragmatique en font autant, les spécialistes des sciences cognitives et de la philosophie analytique également. II est clair qu'il ne sert à rien de tenter de renverser ce genre de mouvement de spécialisation, qui correspond aussi à des mouvements économiques et politiques qui affectent la recherche. En même temps, il semble utile et requis de savoir développer des discours philosophiques plus «traversiers" par rapport à ces sentiers qui deviennent de plus en plus étroits et hermétiques. Ceci est d'autant plus important que la philosophie est régulièrement remise en question dans la culture publique des fonctionnaires et ministres qui en cernent mal la pertinence et l'intérêt.

11. Émile Durkheim, De la division du travail social. Paris, PUF, 1998 (1893), p. 272 ss. Promouvoir une conscience collective de type avant tout technique, ne serait-ce pas valider un type de solidarité tout à fait particulier? En revanche, il est clair qu'aujourd'hui la requête de l'intégration des médias de pointe, que ce soit dans l'enseignement ou ailleurs, fait certes partie des requêtes morales généralisées qui constituent ce que Mead appelait le "generalized other». Voir à ce sujet George H. Mead, Mind, Self and Society. Ch. W. Morris éditeur, Chicago et Londres, University of Chicago Press, 1967 (1934). Mead nous explique entre autres qu'il ne suffit pas, pour que l'individu se développe pleinement comme Soi, qu'il reprenne les attitudes des personnes individuelles envers lui. II doit aussi prendre leurs attitudes "toward the various phases or aspects of the common social activity or set of social undertakings in which, as members of an organized society of social group, they are all engaged (...)", p. 155. L'attitude qui devient obligatoire par rapport à l'Internet et à l'interconnexion en général est de cet ordre.

12. Voir à ce sujet A. Létourneau, L'herméneutique de Maurice Blondel : son émergence pendant la crise moderniste. Montréal, Bellarmin, 1999. 
Une autre composante de notre archaïsme culturel évidemment marquant pour la philosophie, est l'accent porté uniquement sur ce qui est argumentable et donc raisonnable au point parfois d'éclipser d'autres dimensions qui peuvent être vues comme porteuses de sens. Une chose en effet est de reconnaître la valeur et l'importance de la raison et de l'argumentation, passages obligés et lieu normal du travail réflexif pour toute philosophie, une autre chose est de minimiser l'importance des dimensions sensible, émotionnelle ou sentimentale qui caractérisent aussi l'expérience humaine. Cette signification apportée par les sens et l'émotion ne doit pas être considérée comme irrationnelle, mais elle ne doit pas non plus être réduite à ce que nous entendons par la raison de manière générale. Habermas parle des sphères esthétique-expressive, éthiconormative et cognitive instrumentale comme de sphères de rationalité, il ne parle pas pour autant d'une raison esthétique. C'est sans aucun doute sa manière de rendre compte du point que je viens de souligner, quoi que nous puissions estimer qu'il n'accorde peut-être pas assez de développements à cette sphère esthétique-expressive qu'il a au moins le mérite de réhabiliter ${ }^{13}$.

\section{Les problèmes critiques et herméneutiques}

Un premier problème herméneutique surgit précisément du fait que nos principaux outils analytiques sont liés à ces modèles langagiers et écrits dont on a parlé précédemment, ce qui a été bien sûr très important dans les études portant sur le cinéma. L'exemple typique de ce genre d'approche est fourni par les divers courants sémiotiques et structuralistes ${ }^{14}$. Pour le dire en deux mots, l'œuvre est trop foisonnante de sens pour qu'on puisse s'en tenir uniquement à une approche centrée sur un seul de ses aspects, qu'il s'agirait ensuite de décrire en termes de langage de l'œuvre. C'est la complexité même de l'œuvre filmique que rencontre sur son chemin une compréhension trop uniquement fixée sur la dimension langagière du film.

13. J. Habermas, Théorie de l'agir communicationnel. 2 v., Paris, Fayard, 1982. II ne semble pas requis à cet effet de parler d'une "raison esthétique", expression abusive puisque le sens de l'œuvre n'est éventuellement élevé à la "raison" que par une interprétation qui est forcément un décryptage et une reconstruction de son "sens". L'œuvre de Rainer Roschlitz, qui développe cette théorie de la raison esthétique, mérite toutefois sans doute qu'on s'y attarde. Comme le présent texte ne se situe pas au point de vue esthétique mais bien au point de vue herméneutique, ces remarques devront suffire.

14. À partir des années 1960, c'est surtout Christian Metz qui a opéré l'intégration des problématiques sémiologiques et linguistiques dans l'étude du cinéma. Voir en particulier Ch. Metz, Essais sur la signification au cinéma, t. I et II. Paris, Klincksieck, 1968. Analyse syntagmatique, distinction entre le langage cinématographique et le message total de chaque film particulier, intégration des notions saussuriennes de code, signifiant et signifié... 
Le film, en effet, a de nombreuses caractéristiques propres, qui ne se réduisent pas aux aspects langagiers. Mentionnons notamment le recours à l'image, au mouvement, à la proxémie, aux enchaînements de plans, aux scènes, à la diégèse filmique et à l'emphase d'accompagnement audiophonique, ces liens de l'image et du son qui font beaucoup dans les effets de sens du film. Ne faudrait-il pas qu'une interprétation de film tienne compte spécifiquement de la richesse de signification qui est déployée par ces différentes dimensions ou aspects tous interreliés? Cette approche analytique obligerait un découpage plan par plan, scène par scène, séquence par séquence; elle confinerait forcément à l'analyse partielle ${ }^{15}$. Nous pourrions désigner ce premier type d'opération herméneutique comme "décodage horizontal" de la signification. Chaque plan, chaque image peut donner lieu à une lecture analytique rendant compte de la signification en termes de distribution relative des éléments, de proximité et d'associations. Ensuite, le plan peut être replacé dans la série de plans qu'est la scène, puis dans la séquence, ce qui ne correspond plus à l'analyse plan par plan dans la mesure où de nouveaux déplacements et distributions des éléments signifiants interviennent d'une scène à l'autre. L'analyse des séquences pourrait elle-même être considérée comme un premier degré de verticalité introduit dans le film; mais ce n'est pas le seul niveau d'intégration, loin s'en faut puisque la séquence doit elle-même être replacée dans l'ensemble du film. Celui-ci peut à la fois être interprété au plan horizontal (découpage et enchaînement des séquences) et au plan vertical (lecture des oppositions fondamentales qui interviennent dans l'œuvre, herméneutique de la sémiotisation globale).

La précision requise au plan horizontal manifeste la grande richesse sémantique des films. Nous sommes obligés d'opérer des sélections drastiques parmi les matériaux pertinents. Une herméneutique du film doit poser certaines questions bien précises et s'y tenir, c'est sans doute la première condition de son succès. Cette difficulté peut être surmontée simplement par le choix conscient de l'axe de lecture retenu, étant entendu que le critère de décision de la validité d'une interprétation doit être l'œuvre matérielle elle-même; comme disait Eco : si plusieurs lectures d'une œuvre sont légitimes et possibles, certaines d'entre elles ne le sont pourtant pas ${ }^{16}$.

15. Ce qui est assez répandu comme pratique. Voir par exemple Mario J. Valdès, Hermeneutics of Poetic Sense : Critical Studies of Literature, Cinema and Cultural History. Toronto, University of Toronto Press, 1998, p. 52-63 pour ce qui concerne deux films en particulier : Casablanca (1942) et Frida (1984). L'auteur montre en particulier comment l'ouverture du programme narratif concourt à la signification de chaque film.

16. Umberto Eco, Lector in fabula. Paris, Grasset, 1985. - À cet égard, ceci est valide seule- 
II faut avant tout choisir un axe de lecture qui rende compte de l'essentiel de ce que fait en général un film : il raconte une histoire ${ }^{17}$. II faut distinguer entre la diégèse, l'histoire qui est racontée, et la narration, soit la manière qu'a le film de raconter l'histoire. Double lecture qui me semble requise et qui doit avoir le primat sur l'analyse de plans ou de séquences particulières. D'un côté comme de l'autre, la reconstruction sera une écriture, textes de la diégèse et de la narration, ce dernier étant forcément plus analytique que le précédent. Les avantages d'une telle approche au plan pratique sont évidents au point de vue de la lisibilité des œuvres concernées. Elles permettent d'appréhender directement le contenu filmique sur l'un de ses axes, l'axe de la diégèse, en le contrôlant par l'axe de la narration. Pour le dire tout simplement : c'est en racontant le film, dans un récit qui colle évidemment le plus possible aux éléments du médium filmique lui-même, qu'il est possible de donner à son lecteur ou à son auditeur une prise sur l'analyse qui est effectuée. Ceci représente aussi l'avantage de permettre une lecture de l'analyse même pour des personnes qui n'auraient pas vu le film.

$Y$ aurait-il dans cette double écriture une trahison du médium filmique, un enfermement dans le modèle textuel? La difficulté représentée par la prégnance du modèle textuel en herméneutique philosophique semble pouvoir être surmontée, en vertu de deux raisons surtout. D'une part, en fonction de ce que nous avons dit plus haut (la théorie de l'emboîtement des médias), il est toujours possible de traduire un film par un texte, dans la mesure où précisément le film a d'abord été raconté et écrit, scénarisé puis tourné, découpé et monté. Interpréter un film, c'est le traduire en mode réflexif, cette traduction peut être textuelle, tout comme le film a lui-même traduit une série d'intentionnalités fort complexes, qui en partie du moins furent scriptées.

D'autre part, il est certes requis d'intégrer dans l'interprétation du film les outils d'analyse permettant de rendre compte des caractères spécifiques du médium cinéma. Cela demandera en particulier de se familiariser avec le langage technique concerné. Pour pouvoir rendre compte du sens du film, on se croit obligé de passer par les métalangages, or il n'y a pas de métalangage unifié réellement disponible. C'est le problème du foisonnement des métalangages théoriques spécialisés, et de leur non-coordination les uns avec les autres. II s'agit, surtout pour le

ment pour un certain type de films, précisément de genre narratif, qui représente le grand nombre des films. Raul Ruiz a bien montré les limites de cette approche, en particulier concernant le cinéma expérimental, voir Raul Ruiz, Poétique du cinéma. Paris, Dis Voir, 1995.

17. C'est aussi la position d'H.-Paul Chevrier, Le langage narratif du cinéma. Laval, Les 400 coups, 1995. 
novice, d'une véritable Tour de Babel théorique à peu près incompréhensible en dehors du cénacle des spécialistes de tel métalangage en question. C'est une chose en effet de contrôler le langage technique propre à la production du film, entre les plans rapprochés, les séquences et les plans, le cadrage et la composition, c'en est une autre de choisir une grille d'analyse inspirée soit des essais de sémiotique, soit d'autres courants comme la théorie critique, l'approche structurale ou l'approche pragmatique. Deux niveaux de langage qui ont chacun leur spécificité : langage technique du cinéma, et métalangage d'analyse, en termes d'une sémiotique actantielle, en termes narratologiques, etc. ${ }^{18}$ Dans ce contexte, il y a un risque de triplement de l'illisibilité, puisqu'aux difficultés propres du contenu filmique avec les caractéristiques du médium cinéma dans son ensemble, s'ajoutent celles des outils techniques du cinéma et des métalangages auxquels on se réfère. Une difficulté sous-jacente est que forcément, dans le contexte du foisonnement théorique qui a été le fait de l'explosion des analyses structurales puis post-structurales, les interprètes singuliers opèrent des synthèses partielles et personnelles qui sont des hybrides des divers langages théoriques.

En fait, le niveau de réflexion proprement herméneutique ne peut pas se passer d'un certain métalangage et de certains outils théoriques d'analyse bien formés. II doit bien sûr pouvoir maîtriser les catégories de base du médium cinéma. II y a un intérêt manifeste à maîtriser les catégories de base d'une sémiotique, par exemple celle de l'école greimassienne, et/ou de se familiariser avec les approches pragmatique et rhétorique. Ceci étant dit, un questionnement proprement herméneutique doit aller plus loin : il doit avant tout rendre possible un dialogue avec l'œuvre, en faisant ressortir en quoi l'œuvre est question pour nous et notre existence dans le monde, c'est-à-dire ce en quoi elle nous interroge. Elle ne pourra toutefois le faire que si nous sommes en mesure d'expliciter la structure de nos propres attentes de sens, en particulier telles qu'elles seront forcément manifestées par le contenu narratif du film. Dans ce jeu double de la question et de la réponse, notre monde et celui du film pourront mutuellement être éclairés, produisant du sens dans la lecture. Un exemple de stratégie de questionnement qui donne une résonance concrète à cette proposition sera donné plus loin.

18. Pour un exemple d'intégration de l'approche sémiotique de l'école greimassienne dans une perspective herméneutique; voir $A$. Létourneau, "Vers une herméneutique des films". Lecture de 2001: L'odyssée de l'espace (1968), Actes du Colloque A La poétique du divin, Université Laval et Université de Metz, tenu à Québec, en mai 1998, dans R. Hurley (dir.), La poétique du divin. Ste-Foy, Presses de l'Université Laval, 2001, p. 61-86. 
Mais une dimension de la question de l'interprétation de l'œuvre qu'il ne faut absolument pas négliger concerne le moment esthétique de la participation, qui est plus qu'une simple réception de l'œuvre. II est clair que le film, comme l'œuvre d'art en général, a la caractéristique de rendre possible une animation des sentiments de la personne qui le voit. Le spectateur donne vie au film, il le cadre mais aussi est entraîné dans des états émotionnels et moraux qui sont essentiels à sa bonne marche, ces états variant beaucoup selon les films et les spectateurs. L'interprétation du film commence à ce niveau là : dans le mouvement de réception et de participation à la vie du film, où peu de distanciation critique est normalement possible et attendue. Les mauvais films sont justement ceux qui provoquent, sans la rechercher, cette distanciation critique. Certains films ont pu transposer en cinéma le modèle brechtien, mais ce serait alors un effet voulu, donc parfaitement légitime ${ }^{19}$. Voir un bon film, c'est d'ordinaire participer à l'action et aux passions, aux états du sentiment qui sont provoqués par l'enchaînement des plans et des actions. Parfois, la beauté et la noblesse morale des caractères mis en scène provoquent un attachement profond qui élève le sentiment tout en le détachant de la lourdeur qui caractériserait ces sentiments s'ils étaient réellement vécus. Ce que dit Cassirer à propos des arts en général s'applique à cet égard au cinéma ${ }^{20}$. On peut donner à ce sujet l'exemple de Tigre et dragons : la noblesse et la vigueur des sentiments mis en scène, la radicalité des actions entreprises sous le poids de la passion uniquement conviennent parfaitement à la fin tragique du film ${ }^{21}$. Le cadre mythique de l'empire du Milieu et de ses chevaliers, dans le contexte d'arts martiaux appris sous un mode initiatique, se reflète dans les mouvements gracieux des personnages, une chorégraphie où le combat est déréalisé en étant figuré comme une danse. Or si l'effet du film sur le spectateur se mesure en sentiments d'élévation et de noblesse de cœur, c'est bien le cadre

19. Voir Bertolt Brecht, Écrits sur le théâtre. Paris, L'Arche, 1972.

20. Ernst Cassirer, Écrits sur l'art. Paris, Cerf, 1995. Interprétant à sa manière la katharsis, Cassirer précise qu'«ll ne s'agit ni d'une purification ni d'une purge de nos émotions. Cela signifie que nos émotions sont élevées à un nouvel état. Un homme qui, dans la vie réelle, aurait eu à traverser toutes les émotions que nous éprouvons lorsque nous écoutons une tragédie de Sophocle ou de Shakespeare, n'aurait pas été seulement opprimé, mais écrasé et annihilé par le pouvoir de ces émotions. Ce que nous éprouvons ici est la pleine vie des émotions, sans leur contenu matériel. Le fardeau de nos passions est levé de nos épaules; ce qui demeure est le mouvement interne, la vibration et l'oscillation de nos passions, sans leur gravité, leur pression et leur poids", p. 145. Que les partisans de l'esthétique géniale veuillent me pardonner si je rapproche à leur idée indûment dans la suite une œuvre mineure par rapport aux grands classiques du théâtre et de la littérature mondiale!

21. Ang Lee, Tigres et dragons (Hidden Dragon, Crouching Tiger), Sony Pictures, 2000, $120 \mathrm{~m}$. 
mythique lui-même, aisément dégageable, qui les rend possibles. Au plan politique, la noblesse des sentiments vécus vient aussi des nombreuses frontières de classe et de sexe qui sont ici symboliquement renversées; le temps de la mise en récit: entre les femmes et les hommes, puisque les femmes sont ici des combattants de premier plan et des chevaliers au même titre que les hommes, entre les classes sociales aussi puisque les deux principaux rôles féminins, appartenant à des classes opposées, se rapprochent, luttent et ont des destins similaires malgré les différences; la même chose vaut de la relation de la jeune princesse avec Nuage noir, chef de horde sauvage des steppes de l'Extrême-Orient. C'est le Far East qui répond ici au Far West, dans cette production financée par des américains mais dirigée et jouée par des orientaux, notamment Chow Yun Fat et Michelle Yeoh. Le tragique et la beauté du film sont sans doute que l'effort amoureux de cette traversée des classes et des genres menée en toute radicalité conduit à l'échec, qui en relève le caractère de rêve et d'idéal, inaccompli mais persistant. Ce qui, bien sûr, peut aussi permettre un questionnement sur le sentiment amoureux.

Une herméneutique critique qui ne prendrait pas d'abord en compte ce caractère prenant pour l'émotion des participants, la dévaloriserait ou manquerait à la considérer à sa juste place s'échouerait dans le formalisme. En revanche, en rester au sentiment sans vouloir explorer les cadres formels et construits qui le rendent possible serait manquer à une lecture réfléchie des œuvres.

II est clair que depuis quelques décennies, un tournant vers la pragmatique, soit sur l'énonciation comme action plutôt que sur le discours, a été pris 22 . De même, les études spécialisées ont été très marquées par toute l'esthétique de la réception, qui a fait voir l'importance du spectateur dans la production du sens, c'est-à-dire dans l'interprétation elle-même, en particulier à la suite d'Umberto Eco. II semble que c'est dans ce contexte en particulier qu'une herméneutique du film peut apporter une contribution. Je propose qu'elle pose systématiquement des questions à la fois à l'interprète-spectateur, qui est en un sens maître de l'interprétation, et à l'œuvre, en éclairant le monde de l'œuvre par le monde de l'interprète et réciproquement. Plutôt que de se fixer uniquement sur «le sens du film", il s'agirait de rendre possible un dialogue avec l'œuvre. Certaines questions peuvent mener à une discussion porteuse de signification, par exemple dans une classe, en s'adressant en retour aux étudiants.

22. Cf. A. Létourneau, "L'argumentation, la narration et l'affectation". Modalités de l'engendrement pragmatique du discours dans les films Le déclin de l'empire américain (1986) et Jésus de Montréal (1989), à paraître. 
D'abord, il est recommandé de partir de la diégèse et de la narration, d'assurer que le récit a été saisi dans ses composantes propres. Trop souvent en effet, les lectures impressionnistes manquent à saisir la structure propre du récit qui est concerné. Celui-ci est en général assez simple, encore faut-il le dégager convenablement, par exemple : déclenchement, déroulement et résolution de l'intrigue, ce qui a été mis en lumière par la narratologie. Assurer ce premier niveau d'analyse convenablement est indispensable, car il permet d'éviter de passer à côté du film, ou de prétendre y trouver ce qui ne s'y trouve pas. Ceci étant dit sans minimiser l'incontournable pluralisme des interprétations possibles. Celui-ci est d'ailleurs le fait 1) de la très grande multiplicité des éléments mobilisés dans les films et aussi 2) de la pluralité des orientations de valeurs des participants, niveaux qui se mélangent dans la pratique.

Entendons d'abord bien que dans la série de questions que je soumets comme propositions, des sélections peuvent aussi être faites. II n'est sans doute ni nécessaire ni possible de couvrir adéquatement tous ces aspects dans une même séance.

A. 1. Quel genre de monde le film nous donne-t-il à découvrir? 2. Quel genre de société humaine le film présente-t-il? 3. Quelle distribution de rôles humains le film prévoit-il? 4. Comment le film configure-t-il son univers moral, à partir de quels termes clé pouvons-nous en rendre compte? 5. Comment caractériser l'esthétique du film, quelle perception de son univers nous donne-t-il? 6. Quels sentiments donne-t-il à vivre par procuration? 7. Le film dévoile-t-il ou met-il en scène un certain rapport aux configurations mythologiques, religieuses ou métaphysiques qui sont identifiables dans la culture connue?

B. 1. Comment caractériser notre monde? 2. Comment caractériser notre société? 3. Compte tenu du domaine exploré par le film, comment caractériser les dimensions correspondantes dans la vie concrète qui est la nôtre en ce qui concerne la distribution des rôles? 4. Comment caractériser notre univers moral, à partir de quels termes clé, dans la "région" de la culture explorée par le film? 5 . Notre perception du monde et de l'univers, par quelle esthétique la qualifier? 6 . À quelles expériences de sentiment le film renvoie-t-il, y a-t-il des équivalents de ceci dans le monde vécu? 7. Quel est notre propre rapport aux configurations mythiques, religieuses ou métaphysiques qui sont repérables dans le film en étude?

Les questions 1,2 et 3 permettent de dégager, entre autres dimensions, la structure de domination qui est soit directement lisible dans le film, soit y renvoie dans notre propre vie sociale, ou dans celle d'une configuration historique de celle-ci. Les questions 4 et 7 permettent 
en particulier de réfléchir à savoir si et jusqu'à quel point les éléments moraux, religieux ou métaphysiques évoqués dans le film font ou non partie de ce qui peut être vu comme fonction de remplacement de l'idéologie, selon qu'il s'agit ou non d'occurrences de type «Mainstream»23. Les questions 5 et 6 seraient l'espace privilégié du questionnement esthétique, entendant par là aussi bien les effets sur les sentiments que sur la sensibilité, la perception etc. Sans les minimiser, nous devrions situer ces dimensions dans l'ensemble plus vaste représenté par les autres questions. Les questions posées à la section $B$ peuvent sembler très larges, mais il est possible d'en restreindre la portée en se limitant à la discussion de ce qui est présenté par le film comme image du monde, de la société, etc.

Une telle stratégie serait ce que je nommerais une «mise en écho» herméneutique. Le film qui est l'objet d'étude produit une énonciation à propos de certaines choses, c'est un premier présupposé de l'approche. Son énonciation peut être distribuée en principe selon les questions qu'on a dites. Qu'importe de savoir si, en tant qu'œuvre de fiction, l'œuvre n'a qu'un rapport médiat au domaine de la référence, un état réel de choses; il est évident qu'il s'agit d'une création artistique, donc d'une configuration et d'une création autonome ayant son sens propre, mais celle-ci n'aurait aucun effet si elle ne travaillait pas à partir de matériaux ayant une portée soit référentielle, soit esthétique, soit morale sur les personnes, en renvoyant de quelque manière à un monde de la vie 24 . Nous ne sommes pas

23. Voir à ce sujet $\mathrm{A}$. Létourneau, "Les industries culturelles et le problème de la domination : deux approches philosophiques", à paraître dans P.-Y. Bonin (dir.), Philosophie et mondialisation. L'hypothèse du "mainstreaming" renvoie au modèle de la Cultivation Analysis.

24. On aura sans doute reconnu une nouvelle fois la tripartition habermassienne en des énoncés cognitifs-instrumentaux, esthétiques-expressifs et éthico-normatifs. Rendre l'herméneutique des films au domaine de l'interaction communicationnelle, doit être possible même sans préjuger de la validité ou de la complétude du modèle habermassien eu égard aux dimensions normatives de la culture. L'objectif devrait être de pouvoir s'entendre...seulement sur la nécessité de discuter de manière constructive. II semble requis de maintenir, au moins au plan des questions posées, le niveau mythologique et même le niveau des images religieuses ou métaphysiques du monde. D'une part, le plaidoyer habermassien de la relève du religieux dans le contexte de la formation discursive de la volonté souffre sans doute de mésestimer la dimension esthétique et n'épuise pas tout des fonctions culturelles de la religion. II mésestime d'ailleurs beaucoup l'immense récupération des symboliques classiques issues des grandes cultures par l'industrie culturelle. D'autre part, même s'il voyait juste à propos de la "relève", il est certain qu'au point de vue culturel tous n'en sont pas là, loin s'en faut et les industries culturelles vivent du capital symbolique développé dans les grandes cultures, d'où la nécessité d'en tenir compte. Habermas se prononce explicitement sur cette relève par l'agir commmunicationnel, voir TAC II, op. cit., p. 104. II me semble que la perspective cassirérienne a 
obligés de présupposer la "vérité de l'œuvre" au sens de Gadamer : ce serait un trop fort présupposé, contentons-nous d'un dévoilement de monde possible, dont les rapports avec un prétendu réel toujours déjà reconstruit dans le langage articulé sont complexes et peuvent de plus varier énormément d'une œuvre à l'autre ${ }^{25}$. Ne perdons pas de vue d'ailleurs la valeur rhétorique des présentations qui soulignent le caractère "historique» d'un film à partir du fait qu'il s'inspirerait d'un «fait vécu»; ce réalisme filmique est bien sûr lui-même extrêmement construit. La «vérité» toujours relative de l'œuvre ne pourrait advenir que dans la rencontre effective entre l'œuvre et l'interprète.

Le risque inhérent d'une telle approche serait de déporter la discussion au delà du matériel à l'étude, en l'occurrence un film. Mais son avantage serait de donner par le moyen du film un cadre de référence permettant de laisser libre cours à l'imagination, à la pensée et à la parole des interprètes, dans un contexte nettement déterminé par le contenu du film lui-même, mais portant en fin de compte surtout sur le monde que nous vivons. De cette façon, nous passerions concrètement de l'évasion à la discussion. Si par hypothèse le film tourne autour des relations de couples, la discussion serait plutôt limitée à cet aspect. Elle aurait alors pour avantage de rendre possible à la fois une analyse de contenus déterminée, et de ce côté le matériau du film doit pouvoir être analysé en son caractère propre, et de renvoyer au domaine d'objets, de sujets ou de relations qui est celui de l'expérience qu'a tout un chacun de son monde. II devient alors possible de parler de pertinence, d'efficacité ou d'impertinence d'un contenu filmique; on peut aussi relever les questions que le film fait surgir.

\section{Alain Létourneau Professeur agrégé, Université de Sherbrooke}

l'intérêt de reconnaître comme fonction symbolique la dimension mythique de la culture. Pour la critique d'un prétendu égologisme cassirérien, voir le commentaire de Michael $\mathrm{J}$. Krois, Cassirer. Symbolic Forms and History. New Haven, Yale University Press, 1987.

25. Hans Georg Gadamer, Vérité et méthode. Les grandes lignes d'une herméneutique philosophique. Paris, Seuil, 1996. 